\title{
LncRNA CCAT1 negatively regulates miR-181a-5p to promote endometrial carcinoma cell proliferation and migration
}

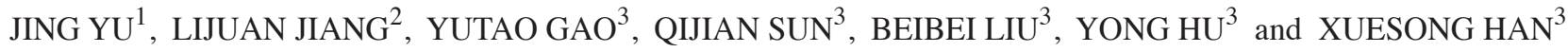 \\ ${ }^{1}$ Department of Gynecology, The Third Affiliated Hospital of Kunming Medical University and Cancer Hospital of \\ Yunnan Province, Kunming, Yunnan 650118; ${ }^{2}$ Department of Scientific Research and Education, \\ The First Affiliated Hospital of Yunnan University of Traditional Chinese Medicine, Kunming, Yunnan 650200; \\ ${ }^{3}$ Department of Gynecology, The First Affiliated Hospital of Kunming Medical University, Kunming, Yunnan 650032, P.R. China
}

Received May 29, 2018; Accepted October 3, 2018

DOI: $10.3892 /$ etm.2019.7422

\begin{abstract}
Long non-coding RNAs (lncRNAs) are identified as vital modulators in a number of biological processes, including tumorigenesis. However, the role of lncRNAs in endometrial carcinoma (EC) remains unknown. In the current study, the expression patterns, biological roles and functional mechanism of the IncRNA colon cancer associated transcript 1 (CCAT1) was examined in EC. The expression level of CCAT1 was significantly upregulated in EC tissue samples compared with matched adjacent healthy tissue samples from patients with endometrial cancer. Similarly, CCAT1 was significantly upregulated in several EC cell lines (KLE, Ishiwaka and HEC-1-A), compared with the normal human endometrial stromal cell line T-HESC. Cell counting kit- 8 and Transwell migration assays demonstrated that CCAT1 knockdown significantly decreased EC cell proliferation and migration. In addition, CCAT1 was confirmed as a target gene of miR-181a-5p in EC. Overexpression of miR-181a-5p significantly decreased CCAT1 expression in EC cells, whilst knockdown of CCAT1 significantly increased miR-181a-5p expression in EC cells. Furthermore, miR-181a-5p expression was significantly downregulated in EC tissue samples compared with matched adjacent healthy tissue samples from patients with endometrial cancer. Similarly, miR-181a-5p expression was significantly downregulated in several EC cell lines (KLE, Ishiwaka and HEC-1-A), compared with normal human endometrial stromal cell line T-HESC. In addition, rescue experiments demonstrated that inhibition of miR-181a-5p significantly reversed the effect of CCAT1 knockdown on EC cell proliferation and migration. The results suggest that CCAT1 promotes EC progression by acting as a molecular sponge of miR-181a-5p.
\end{abstract}

Correspondence to: Professor Xuesong Han, Department of Gynecology, The First Affiliated Hospital of Kunming Medical University, 295 Xichang Road, Kunming, Yunnan 650032, P.R. China E-mail: hanxuesongzg@163.com

Key words: long noncoding RNA, endometrial carcinoma, colon cancer associated transcript 1 , proliferation, migration

\section{Introduction}

Endometrial carcinoma (EC) is one of the most common malignancies in women, and a leading cause of cancer-associated mortality worldwide (1). The most frequently occurring subtype of endometrial cancer is endometrioid endometrial carcinoma, which accounts for about $90 \%$ of all EC cases (2). Surgery is the main treatment strategy for patients with EC (3), however the effectiveness of primary surgery is generally unsatisfactory (3). Although the incidence of EC is increasing, the molecular mechanism underlying EC progression remains poorly understood (2). It is therefore necessary to explore the mechanism and develop novel therapeutic strategies for EC intervention.

Long non-coding RNAs (lncRNAs) are a large and diverse class of transcribed RNA molecules usually with a length of more than 200 nucleotides and limited protein-coding capability (4). LncRNAs can regulate gene expression via several different mechanisms, which include acting as a molecular sponge for miRNAs to regulate the expression of its target genes (5). Several studies have demonstrated that lncRNAs are involved in a number of biological processes, including tumor initiation, growth and metastasis $(6,7)$. The lncRNA gastric cancer associated transcript 3 promotes colorectal cancer cell proliferation, invasion and migration through competitive binding to miRNA-149 (8). LncRNA long intergenic non-protein coding RNA 1510 promotes colorectal cancer cell growth by modulating MET expression (9). LncRNA FLVCR1-antisense RNA 1 acts as a miRNA-513c sponge to regulate cancer cell proliferation, migration and invasion in hepatocellular carcinoma (10). LncRNA long intergenic non-protein coding RNA 958 accelerates gliomagenesis through miRNA-203/cyclin-dependent kinase 2 regulation (11).

A recently study revealed that IncRNA colon cancer associated transcript 1 (CCAT1) promotes cell proliferation, migration and invasion in thyroid carcinoma via downregulation of miRNA-143 (12). Other studies have demonstrated that CCAT1 is involved in colorectal cancer (13), ovarian cancer (14), gastric cancer (15), myeloma (16) and breast cancer (17). The pathophysiological contribution of CCAT1 to EC progression remains largely unknown. The aim of the 
present study was to investigate the biological function of CCAT1 in EC. The current study demonstrated that CCAT1 was significantly upregulated in EC tissue samples and cell lines. Knockdown of CCAT1 significantly decreased EC cell proliferation and migration. Furthermore, the current study demonstrated that CCAT1 acts as a molecular sponge of miRNA-181a-5p (miR-181a-5p) in EC cells and inhibition of miR-181a-5p significantly reversed the effects of CCAT1 knockdown. Taken together, the current study revealed that the CCAT1/miR-181a-5p axis may serve a role in EC progression.

\section{Materials and methods}

Patient samples. The present study analyzed tissue samples from patients diagnosed with endometrial cancer based on histopathological evaluation. A total of $37 \mathrm{EC}$ tissue and 21 matched adjacent normal tissue samples were collected from female patients (mean age, $47.56 \pm 15.49$ years) who had undergone surgical resection at The First Affiliated Hospital of Kunming Medical University between January 2010 and December 2016. None of the patients received local or systemic treatment before the operation. Endometrial cancer tissue and adjacent healthy tissue samples were collected and immediately frozen in liquid nitrogen, and stored at $-80^{\circ} \mathrm{C}$ until further use. The current study was approved by the Ethics Committee of The First Affiliated Hospital of Kunming Medical University (Yunnan, China). Written informed consent was obtained from all patients.

Cell culture and transfection. Human endometrial adenocarcinoma cell lines HEC-1-A (ATCC ${ }^{\circledR}$ HTB-112 ${ }^{\mathrm{TM}}$ ) and KLE (ATCC ${ }^{\circledR}$ CRL-1622 ${ }^{\mathrm{TM}}$ ), as well as the human endometrial stromal cell line T-HESC (ATCC ${ }^{\circledR}$ CRL-4003 ${ }^{\mathrm{TM}}$ ) were from American Type Culture Collection (Manassas, VA, USA). Human endometrial adenocarcinoma cell line Ishikawa (cat. no. 3111C0001CCC000686) was purchased from China Infrastructure of Cell Line Resource (Beijing, China; http://www.cellresource.cn/contact.aspx). All cell lines were maintained in Dulbeco's modified Eagle medium (DMEM; Invitrogen; Thermo Fisher Scientific, Inc., Waltham, MA, USA) supplemented with $10 \%$ fetal bovine serum (FBS; HyClone; GE healthcare, Chicago, IL, USA) and $1 \%$ penicillin-streptomycin solution and maintained at $37^{\circ} \mathrm{C}$ in a $5 \% \mathrm{CO}_{2}$-humidified incubator. miR-181a-5p mimic (5'-AACAUUCAACGCUGUCGGUGAGU-3'), miR-181a-5p inhibitor (5'-ACUCACCGACAGCGUUGAAUGUU-3'), negative controls miRNA (5'-UCACAACCUCCUAGA AAGAGUAGA-3'), si-CCAT1 (5'-AGGGAAACAGGAGCA AUCAUCATTA-3') and control siRNA (5'-UUCUCCGAA CGUGUCACGUTT-3') were chemically synthesized by Shanghai Integrated Biotech Solutions Co., Ltd. (Shanghai, China). All cell transfection reactions were performed using Lipofectamine $^{\circledR} 2000$ (Invitrogen; Thermo Fisher Scientific, Inc.) with $100 \mathrm{nM}$ miRNA or siRNA, according to the manufacturer's protocol. Subsequent experiments were performed following $48 \mathrm{~h}$ transfection.

Reverse transcription-quantitative polymerase chain reaction $(R T-q P C R)$. Total RNA was isolated from tissues or cultured cells using TRIzol ${ }^{\circledR}$ reagent (Invitrogen; Thermo Fisher
Scientific, Inc.), according to the manufacturer's protocol. Total RNA $(5 \mu \mathrm{g})$ was reverse transcribed into cDNA using M-MLV Reverse Transcriptase (Invitrogen; Thermo Fisher Scientific, Inc.) and supplemented with Oligo(dT18) RT primers (Invitrogen; Thermo Fisher Scientific, Inc.). qPCR was subsequently performed using SYBR Premix Ex Taq (Takara Bio, Inc., Otsu, Japan) using a Bio-Rad CFX96 real-time PCR System (Bio-Rad Laboratories, Inc., Hercules, CA, USA). The conditions of qPCR were as follows: $94^{\circ} \mathrm{C}$ for $15 \mathrm{~min}$, followed by 45 cycles of $94^{\circ} \mathrm{C}$ for $10 \mathrm{sec}, 60^{\circ} \mathrm{C}$ for $30 \mathrm{sec}$, and $72^{\circ} \mathrm{C}$ for $30 \mathrm{sec}$. Each sample assayed in triplicate in three independent experiments. The mRNA levels were quantified using the $2^{-\triangle \Delta C q}$ method (18) and normalized to internal reference gene U6. The sequence of the CCAT1 forward primer was 5'-TTTATGCTTGAGCCTTGA-3' and reverse primer was 5'-CTTGCCTGAAATACTTGC-3'. The sequence of the miR-181a-5p forward primer was 5'-GCCGAACATTCAACG CTGTCG-3' and reverse primer was 5'-GTGCAGGGTCCG AGGT-3'. The sequence of the miR-181a-5p forward primer was 5'-CTCGCTTCGGCAGCACA-3' and reverse primer was 5'-AACGCTTCACGAATTTGCGT-3'. The sequence of U6 forward primer was 5'-CTCGCTTCGGCAGCACA-3' and reverse primer was 5'-AACGCTTCACGAATTTGCGT-3'.

Cell proliferation assay. Cell counting kit-8 (CCK-8; Beyotime Institute of Biotechnology, Haimen, China) was used to study cell proliferation. KLE cells were seeded into 96-well plates at a density of $1 \times 10^{3}$ cells/well and cultured for 24,48 or $72 \mathrm{~h}$. Cells were subsequently incubated with $10 \mu \mathrm{l}$ of CCK- 8 for $2 \mathrm{~h}$ at $37^{\circ} \mathrm{C}$. Following incubation with $\mathrm{CCK}-8$, cell viability was determined by measuring the absorbance at a wavelength of $450 \mathrm{~nm}$.

Cell migration assay. Cell migration assays were performed using $8 \mu \mathrm{m}$ transwell inserts (EMD Millipore, Billerica, MA, USA). A total of $2 \times 10^{4} \mathrm{KLE}$ cells were added to the top chamber containing $200 \mathrm{ml}$ serum-free DMEM. DMEM supplemented with $10 \%$ FBS was added to the lower chamber. After $48 \mathrm{~h}$ incubation, migrated cells in the lower chamber were stained with $0.1 \%$ crystal violet for $1 \mathrm{~h}$ at $25^{\circ} \mathrm{C}$. Using a light microscope at x100 magnifications, five visual fields were randomly selected to calculate the number of migrated cells.

Western blot analysis. Total protein was extracted from KLE cells using radioimmunoprecipitation lysis buffer (Beyotime Institute of Biotechnology). Total protein was quantified using a bicinchoninic acid assay kit and $40 \mu \mathrm{g}$ protein/lane was separated via SDS-PAGE on a $8-12 \%$ gel. The separated proteins were subsequently transferred onto polyvinylidene fluoride membranes and blocked with $5 \%$ non-fat milk for $1 \mathrm{~h}$ at $37^{\circ} \mathrm{C}$. The membranes were incubated with primary antibodies against GAPDH (1:5,000; cat. no. SAB2701826; Sigma-Aldrich; Merck KGaA, Darmstadt, Germany) and c-MET (1:2,000; cat. no. ab51067; Abcam, Cambridge, UK) overnight at $4^{\circ} \mathrm{C}$. Following primary incubation, the membranes were incubated with a goat horseradish peroxidase-conjugated secondary antibody (1:5,000; sc-2005; Santa Cruz Biotechnology, Inc., Dallas, TX, USA) for $2 \mathrm{~h}$ at room temperature. Protein bands were visualized using enhanced chemiluminescence substrate (EMD Millipore). 
Bioinformatics analysis. The target miRNAs of CCAT1 were predicted using miRDB (http://mirdb.org/miRDB/index.html).

Luciferase reporter assay. A luciferase reporter assay was performed to determine the direct binding of CCAT1 to miR-181a-5p. The wild type (WT) and mutant (Mut) sequence of CCAT1 was directly synthesized by Shanghai GenePharma Co., Ltd. (Shanghai, China) and then inserted into pGL3 plasmid (Ambion; Thermo Fisher Scientific, Inc.). Cells $\left(2 \times 10^{4}\right)$ were cultured in 24-well plates, and each well was transfected with $0.2 \mu \mathrm{g}$ WT or Mut pGL3-CCAT1 reporter plasmid, and equal amounts of miR-181a-5p (50 nM) and NC mimics (50 nM) using Lipofectamine ${ }^{\circledR} 2000$ (Invitrogen; Thermo Fisher Scientific, Inc.). After 24 h, Firefly and Renilla luciferase activities were measured using the Dual-Luciferase Reporter Assay Kit (Promega Corporation, Madison, WI, USA) according to the manufacturer's protocols. Luciferase activities were normalized to that of Renilla luciferase.

Statistical analysis. Data are presented as the mean \pm standard deviation of three independent experiments. All statistical analyses were performed using SPSS software (version 20.0; IBM Corp., Armonk, NY, USA). Student's t-test was performed for comparison analysis between two groups. One-way analysis of variance followed by Fisher's least significant difference post hoc test was performed for multiple-group comparisons. Pearson's correlation coefficient was used to measure the linear correlation between CCAT1 and miR-181a-5p expression in EC tissues. $\mathrm{P}<0.05$ was considered to indicate a statistically significant difference.

\section{Results}

Expression patterns of CCAT1 and miR-181a-5p in EC tissues. To investigate the role of CCAT1 in endometrial cancer progression, CCAT1 expression was examined in tissue samples from patients with endometrial cancer. The expression level of CCAT1 was significantly increased in endometrial cancer tissue samples compared with matched adjacent healthy tissue samples from patients with endometrial cancer (Fig. 1A). Similarly, the expression level of CCAT1 significantly increased in several endometrial cancer cell lines, compared with normal human endometrial stromal cell line T-HESC (Fig. 1B). By contrast, miR-181a-5p expression was significantly decreased in endometrial cancer tissue samples compared with matched adjacent healthy tissue samples from patients with endometrial cancer (Fig. 1C). Similarly, the expression level of miR-181a-5p significantly decreased in several endometrial cancer cell lines, compared with normal human endometrial stromal cell line T-HESC (Fig. 1D). These results suggest that CCAT1 may regulate miR-181a-5p expression in EC.

CCAT1 is a target of miR-181a-5p. To investigate the correlation between CCAT1 and miR-181a-5p, bioinformatics analysis was performed using starBase (starbase.sysu.edu.cn) to identify potential targets of miR-181a-5p. starBase identified a potential miR-181a-5p binding site in CCAT1 (Fig. 2A). Luciferase reporter gene assay was performed to confirm the potential association between CCAT1 and miR-181a-5p. Following transfection with miR-181a-5p mimic, the overexpression of miR-181a-5p was confirmed by RT-qPCR (Fig. 2B). Overexpression of miR-181a-5p significantly reduced the luciferase reporter activity of CCAT1-WT compared with CCAT1-Mut in KLE cells (Fig. 2B). Following transfection with siCCAT1, knockdown of CCAT1 expression was confirmed by RT-qPCR (Fig. 2C). Knockdown of CCAT1 significantly increased miR-181a-5p expression in KLE cells (Fig. 2D) By contrast, overexpression of miR-181a-5p significantly reduced CCAT1 expression in KLE cells (Fig. 2E). Furthermore, an inverse correlation was observed between miR-181a-5p and CCAT1 expression in EC tissues (Fig. 2F). These results suggest that CCAT1 can act as a molecular sponge of miR-181a-5p in EC cells.

CCAT1 knockdown inhibits EC cell proliferation and migration. The physiological functions of CCAT1 in EC were further investigated. CCK-8 assays demonstrated that knockdown of CCAT1 significantly inhibited the proliferation of KLE cells (Fig. 3A). The association between CCAT1 and migration was examined by Transwell migration assays. The results from the migration assays demonstrated that knockdown of CCAT1 significantly decreased the migration ability of KLE cells (Fig. 3B).

miR-181a-5p overexpression inhibits EC cell proliferation and migration. The present study demonstrated that CCAT1 regulates miR-181a-5p expression in EC cells. To determine the physiological function of miR-181a-5p in EC, KLE cells were transfected with miR-181a-5p mimic and subsequent CCK-8 and transwell assays were performed. Overexpression of miR-181a-5p significantly reduced EC cell proliferation and migration (Fig. 4A and B), suggesting that miR-181a-5p may function as a tumor suppressor in EC.

miR-181a-5p inhibition significantly reverses the effects of CCAT1 knockdown in EC cells. To determine whether CCAT1 functions in EC progression through the inhibition of miR-181a-5p, rescue experiments were performed in EC cells. Following transfection with miR-181a-5p inhibitor alone, the relative expression level of miR-181a-5p in KLE cells was confirmed by RT-qPCR (Fig. 5A). CCK-8 and Transwell migration assays demonstrated that CCAT1 knockdown significantly inhibits the proliferation and migration of KLE cells, however these effects were reversed by the inhibition of miR-181a-5p (Fig. 5B and C). These results suggest that CCAT1 may act as a sponge of miR-181a-5p to exert oncogenic roles by regulating EC cell proliferation and migration.

miR-181a-5p targets $c$-MET in KLE cells. miR-181a-5p was previously reported to target c-MET in liver cancer (19). Therefore, the effect of miR-181a-5p on c-MET expression in KLE cells was evaluated. Western blotting demonstrated that miR-181a-5p mimic transfection suppressed c-MET expression in KLE cells (Fig. 6A). Furthermore, CCAT1 knockdown led to decreased expression of c-MET (Fig. 6B), indicating that the CCAT1/miR-181a-5p axis may regulate c-MET to exert roles in EC progression. 

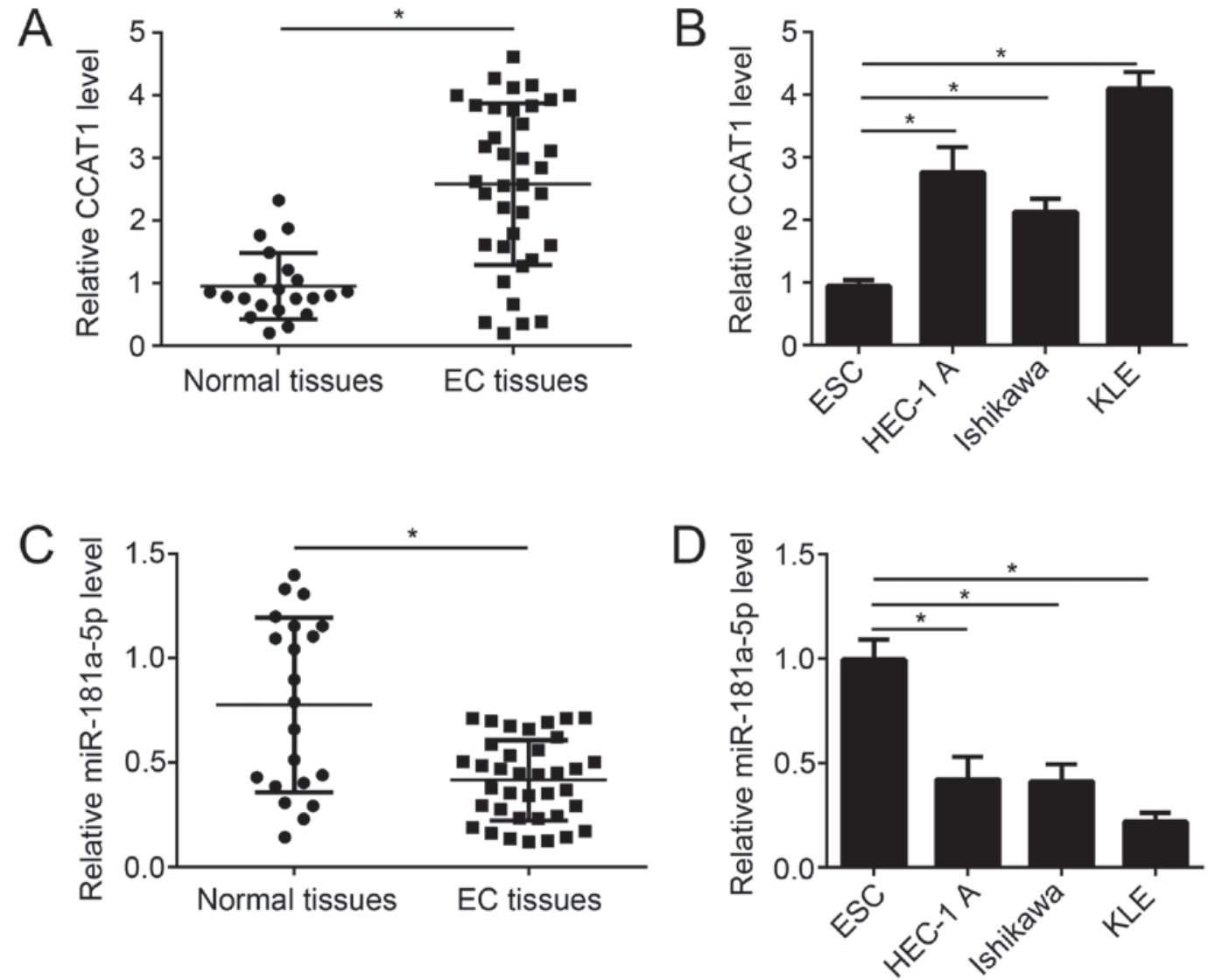

Figure 1. Expression patterns of CCAT1 and miR-181a-5p in EC tissues. (A) The mRNA expression level of CCAT1 was determined by RT-qPCR using tissue samples from patients with endometrial cancer. (B) The relative mRNA expression of CCAT1 was determined by RT-qPCR in human endometrial stromal cell line ESC as well as several human endometrial adenocarcinoma cell lines (HEC-1 A, Ishiwaka and KLE). (C) The mRNA expression level of miR-181a-5p was determined by RT-qPCR using tissue samples from patients with endometrial cancer. (D) The relative mRNA expression of miR-181a-5p was determined by RT-qPCR in human endometrial stromal cell line ESC as well as several human endometrial adenocarcinoma cell lines (HEC-1 A, Ishiwaka and KLE). ${ }^{*} \mathrm{P}<0.05$ as indicated. RT-qPCR, reverse transcription-quantitative polymerase chain reaction; CCAT1, colon cancer-associated transcript 1; EC, endometrial cancer; miR, microRNA; ESC, human endometrial stromal cell line; HEC-1-A, Ishiwaka and KLE, human endometrial adenocarcinoma cell lines.
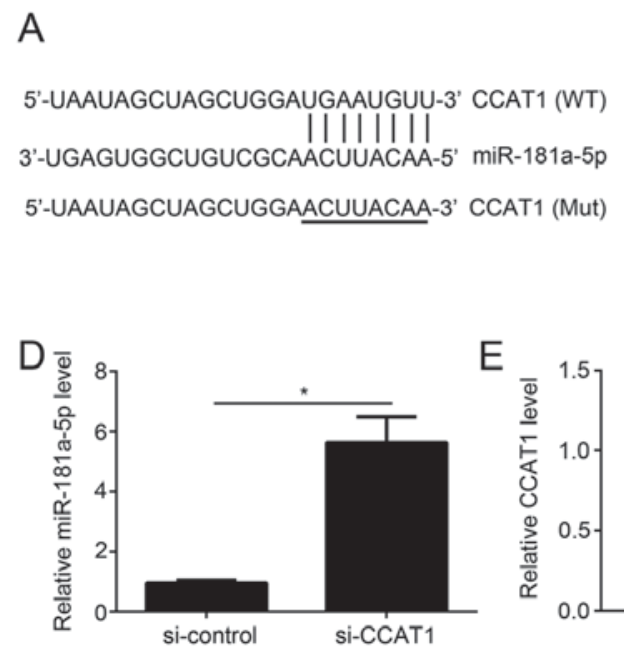
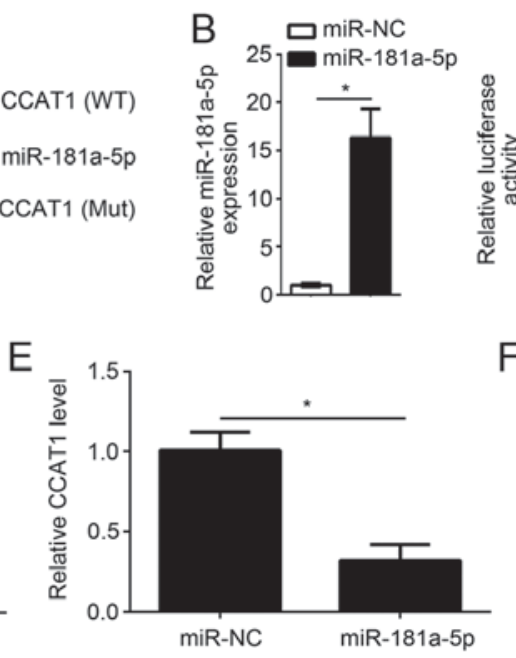
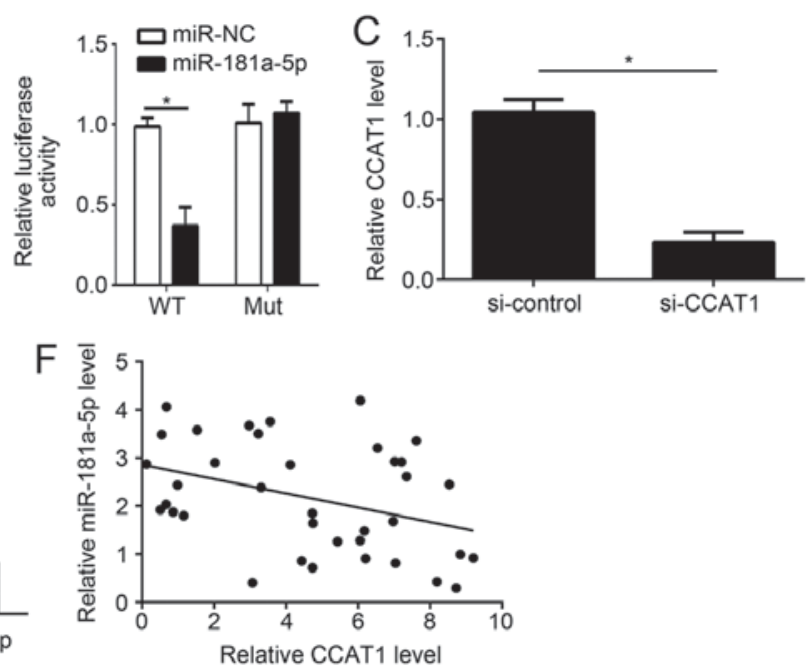

Figure 2. CCAT1 is a target gene of miR-181a-5p. (A) Bioinformatics analysis was used to identify miR-181a-5p binding sites in CCAT1. (B) Following miR-181a-5p overexpression, the expression level was detected by RT-qPCR and the relative luciferase activity was measured. (C) Following transfection with si-CCAT1 or control, the mRNA expression level of CCAT1 was determined by RT-qPCR in human EC cell line, KLE. (D) CCAT1 knockdown significantly enhanced miR-181a-5p expression in KLE cells. (E) Overexpression of miR-181a-5p significantly reduced the mRNA expression level of CCAT1 in KLE cells. (F) An inverse correlation between CCAT1 and miR-181a-5p expression in EC tissue samples was identified ( $\mathrm{r}=-0.371$; $\mathrm{P}=0.024)$. $\mathrm{P}<0.05$ vs. control group. RT-qPCR, reverse transcription-quantitative polymerase chain reaction; CCAT1, colon cancer-associated transcript 1 ; EC, endometrial cancer; miR, microRNA; KLE, human endometrial stromal cell line; miR-NC, endometrial cancer cell line KLE transfected with scramble miR; miR-181a-5p, endometrial cancer cell line KLE transfected with miR-181a-5p mimic; si-control, endometrial cancer cell line KLE transfected with siRNA negative control; si-CCAT1, endometrial cancer cell line KLE transfected with siRNA targeting CCAT1; siRNA, small interfering RNA; CCAT1 (WT), wild-type 3'UTR of CCAT1 cloned into luciferase reporter plasmid; CCAT1 (Mut), mutant 3'UTR of CCAT1 cloned into luciferase reporter plasmid. 

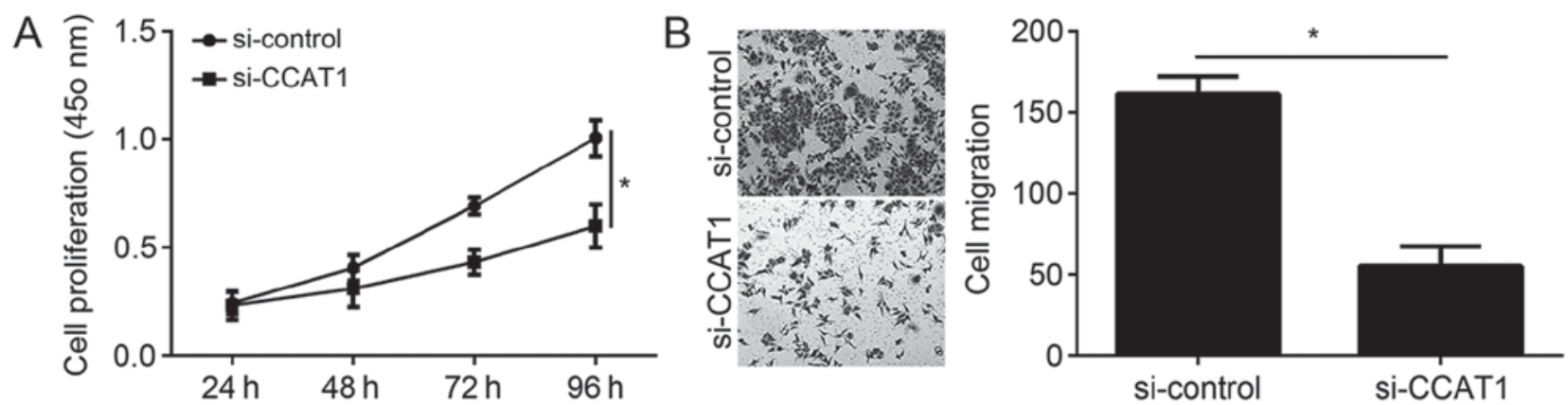

Figure 3. CCAT1 knockdown inhibits EC cell proliferation and migration. (A) The effect of CCAT1 knockdown on KLE cell proliferation was analyzed by CCK-8 assay. (B) The effect of CCAT1 knockdown on KLE cell migration was analyzed using the Transwell migration assay (original magnification, x100). ${ }^{*} \mathrm{P}<0.05$ vs. control group. CCK-8, cell counting kit-8; CCAT1, colon cancer-associated transcript 1; EC, endometrial cancer; miR, microRNA; KLE, human endometrial stromal cell line; si-control, endometrial cancer cell line KLE transfected with siRNA negative control; si-CCAT1, endometrial cancer cell line KLE transfected with siRNA targeting CCAT1; siRNA, small interfering RNA.
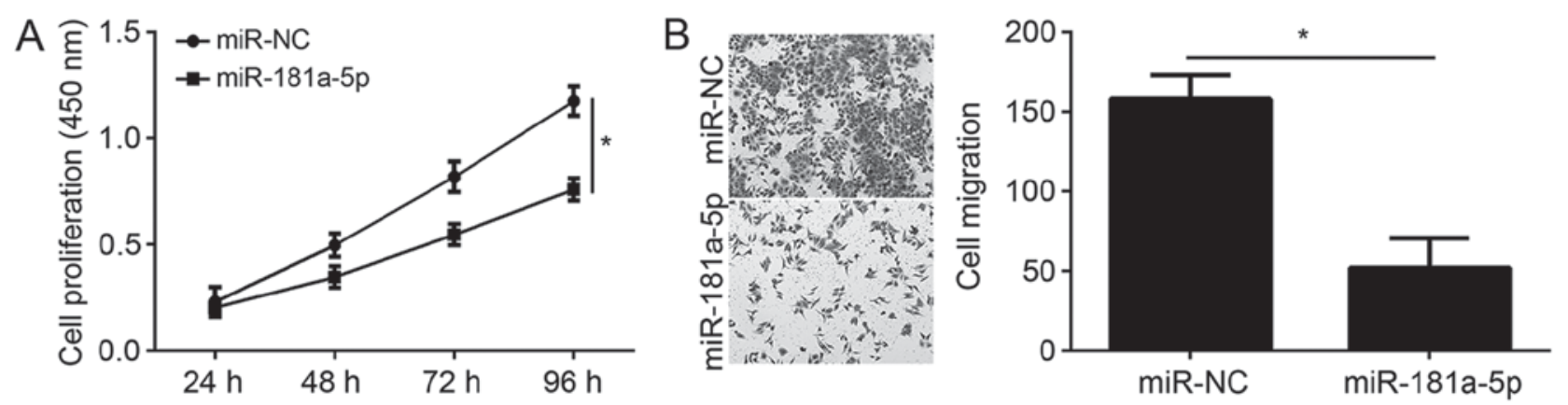

Figure 4. miR-181a-5p overexpression inhibits EC cell proliferation and migration. (A) The effect of miR-181a-5p overexpression on KLE cell proliferation was analyzed by CCK-8 assay. (B) The effect of miR-181a-5p overexpression on KLE cell migration was analyzed using the Transwell migration assay. "P $<0.05$ vs. control group. CCK-8, Cell Counting Kit-8; KLE, human endometrial stromal cell line; miR, microRNA; miR-NC, endometrial cancer cell line KLE transfected with scramble miR; miR-181a-5p, endometrial cancer cell line KLE transfected with miR-181a-5p mimic.

\section{Discussion}

Endometrial cancer is one of the most common malignancies in women, and a leading cause of cancer-associated mortality worldwide (3). Dysregulated expression of lncRNAs is closely associated with tumor development and progression (20). In addition, IncRNAs have been identified as promising biomarkers for assessing cancer diagnosis and prognosis (21). Wang et al (22) reported that upregulation of lncRNA HNF1A-antisense RNA 1 is associated with poor prognosis in bladder cancer. Yang et al (23) demonstrated that lncRNA small nucleolar RNA host gene 1 can predict a poor prognosis and promote colon cancer tumorigenesis. LncRNA BDNF-antisense RNA has functional role in human prostate cancer and is associated with clinical outcomes (24). Xiao et al (25) revealed that lncRNA HOX transcript antisense RNA can be used as a prognostic biomarker for the proliferation and chemoresistance of colorectal cancer via miRNA-203a-3p-mediated Wnt/3-catenin signaling pathway.

The pathophysiological function of lncRNAs in EC requires further investigation. Previous studies have revealed that CCAT1 may serve important roles in the progression and metastasis of cancer. For example, Cao et al (26) indicated that CCAT1 promotes metastasis and poor prognosis in epithelial ovarian cancer. Zhang et al (27) demonstrated that the CCAT1/miRNA-218/zinc finger protein X-linked axis regulates the progression of laryngeal squamous cell cancer. In addition, the CCAT1 promotes osteosarcoma proliferation and migration via regulating the miRNA-148a/phosphoinositide-3-kinase interacting protein 1 signal pathway (28). Arunkumar et al (29) revealed that CCAT1 is overexpressed in oral squamous cell carcinoma and as CCAT1 overexpression may sponge miRNA15-5p and let7b-5b, CCAT1 overexpression may predict poor prognosis. The role of CCAT1 has not been defined in EC progression. The present study demonstrated that CCAT1 expression was significantly upregulated in endometrial cancer tissue samples compared with matched adjacent healthy tissue samples from patients with endometrial cancer. Similarly, CCAT1 expression was significantly upregulated in several EC cell lines (KLE, Ishiwaka and HEC-1-A), compared with normal human endometrial stromal cell line T-HESC. CCK-8 and Transwell migration assays verified that CCAT1 knockdown significantly decreased EC cell proliferation and migration, suggesting that CCAT1 may contribute to EC progression.

Growing evidence has suggested that lncRNAs could act as miRNA sponges to exert a physiological function (30). Xu et al (31) demonstrated that IncRNA differentiation antagonizing non-protein coding RNA acts as a sponge of miRNA-634 to regulate Ras-related protein Rab-1A expression in glioma. Zhu et al (32) revealed that lncRNA taurine up-regulated 1 acts as a sponge of miRNA-138-5p to regulate sirtuin 1 expression 

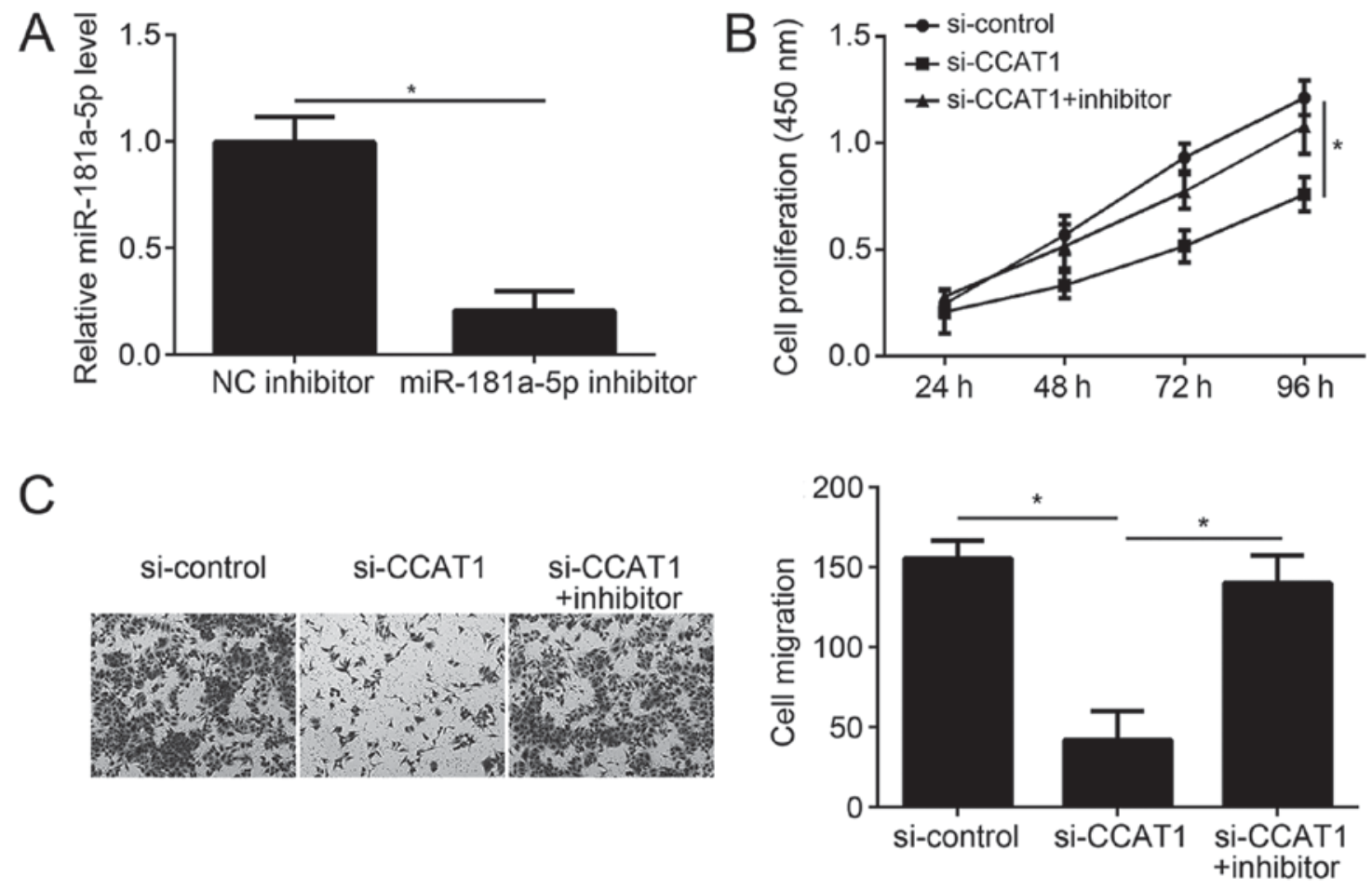

Figure 5. miR-181a-5p inhibition significantly reverses the effects of CCAT1 knockdown in EC cells. (A) Following miR-181a-5p inhibition, the relative expression level of miR-181a-5p was detected in KLE cells by reverse transcription-quantification polymerase chain reaction. (B) The CCK-8 assay was used to analyze the effect of CCAT1 knockdown and miR-181a-5p inhibition on KLE cell proliferation. (C) The Transwell migration assay was used to analyze the effect of CCAT1 knockdown and miR-181a-5p inhibition on KLE cell migration. " $\mathrm{P}<0.05$ as indicated. CCAT1, colon cancer-associated transcript 1; EC, endometrial cancer; miR, microRNA; KLE, human endometrial stromal cell line; NC, negative control; NC inhibitor, endometrial cancer cell line KLE transfected with negative control inhibitor; miR-181a-5p inhibitor, endometrial cancer cell line KLE transfected with miR-181a-5p inhibitor; si-control, endometrial cancer cell line KLE transfected with siRNA negative control; si-CCAT1, endometrial cancer cell line KLE transfected with siRNA targeting CCAT1; siRNA, small interfering RNA; si-CCAT1 + inhibitor, endometrial cancer cell line KLE transfected with siRNA targeting CCAT1 and miR-181a-5p inhibitor.
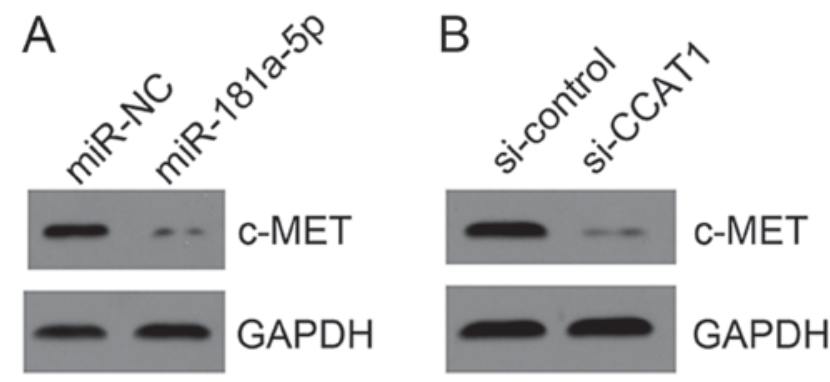

Figure 6. miR-181a-5p targets c-MET in KLE cells. (A) Following miR-181a-5p overexpression in KLE cells, the protein expression level of c-MET was determined by western blotting. (B) Following CCAT1 knockdown in KLE cells, the protein expression level of c-MET was determined by western blotting. CCAT1, colon cancer-associated transcript 1; miR, microRNA; KLE, human endometrial stromal cell line; miR-NC, endometrial cancer cell line KLE transfected with scramble miR; miR-181a-5p, endometrial cancer cell line KLE transfected with miR-181a-5p mimic; si-control, endometrial cancer cell line KLE transfected with siRNA negative control; si-CCAT1, endometrial cancer cell line KLE transfected with siRNA targeting CCAT1; siRNA, small interfering RNA; c-MET, MET proto-oncogene, receptor tyrosine kinase.

and cervical cancer progression. Chen et al (16) revealed that CCAT1 can promote multiple myeloma progression by acting as a molecular sponge of miR-181a-5p and thereby regulating homeobox A1 expression. Additionally, previous studies have demonstrated that IncRNA expression may be regulated by the binding of sponged miRNAs $(33,34)$. Consistent with previous findings, the present study identified that CCAT1 acts as a molecular sponge of miR-181a-5p to suppress miR-181a-5p expression in EC cells. Previous studies have revealed that miR-181a-5p regulates the progression of several types of cancer. Li et al (35) demonstrated that nuclear paraspeckle assembly transcript 1 promotes proliferation and invasion via targeting of miR-181a-5p in non-small cell lung cancer. Additionally, miR-181a-5p promotes the proliferation and migration of gastric cancer cells through regulation of protein tyrosine phosphatase, non-receptor type 9 (36). Yang et al (37) indicated that miR-181a-5p promotes proliferation and invasion, and inhibits apoptosis of cervical cancer cells via targeting of inositol polyphosphate-5-phosphatase A. The role of miR-181a-5p in EC has not been previously investigated. The present study demonstrated that miR-181a-5p expression was significantly downregulated in endometrial cancer tissue samples compared with matched adjacent healthy tissue samples from patients with endometrial cancer. Similarly, miR-181a-5p expression was significantly downregulated in several EC cell lines (KLE, Ishiwaka and HEC-1-A), compared with normal human endometrial stromal cell line T-HESC. CCK-8 and Transwell migration assays demonstrated that miR-181a-5p overexpression inhibited EC cell proliferation and migration. Furthermore, rescue experiments revealed that inhibition of miR-181a-5p significantly reversed the effects of CCAT1 knockdown on EC cell proliferation and migration. A previous study demonstrated that miR-181a-5p was downregulated and suppressed motility, invasion and branching-morphogenesis 
by directly targeting c-MET in liver cancer (19). Additinally, c-MET signaling is crucial in the development of EC (38). The present study revealed that either miR-181a-5p overexpression or CCAT1 knockdown decreased c-MET expression in EC cells, suggesting CCAT1 may be involved in EC progression via c-MET signaling. Furthermore, miR-181a-5p may also be sponged by other lncRNAs, including NEAT1 (35). CCAT1 may function with other lncRNAs to sponge miR-181a-5p and regulate EC progression, however this requires futher investigation.

In conclusion, the current study demonstrated that CCAT1 may function as an oncogene in EC by acting as a molecular sponge of miR-181a-5p. These results suggest a potential molecular mechanism underlying tumorigenesis in EC and the CCAT1/miR-181a-5p axis may be a promising target for EC therapy.

\section{Acknowledgements}

Not applicable.

\section{Funding}

The present study was supported by the Natural Science Foundation of China (grant no. 81560785).

\section{Availability of data and materials}

All datasets used and/or analyzed during the current study are available from the corresponding author upon reasonable request.

\section{Authors' contribution}

JY and XH designed the study and analyzed the data. XH wrote the manuscript. LJ, YG, QS, BL and YH performed the experiments. All authors read and approved the final manuscript.

\section{Ethics approval and consent to participate}

The present study was approved by the Institutional Ethics Committee of The First Affiliated Hospital of Kunming Medical University (Yunnan, China). Written informed consent was obtained from all participants.

\section{Patient consent for publication}

Patients have provided written informed consent for publication of their data.

\section{Competing interests}

The authors declare that they have no competing interests.

\section{References}

1. Felix AS, Scott McMeekin D, Mutch D, Walker JL, Creasman WT, Cohn DE, Ali S, Moore RG, Downs LS, Ioffe OB, et al: Associations between etiologic factors and mortality after endometrial cancer diagnosis: The NRG Oncology/Gynecologic Oncology Group 210 trial. Gynecol Oncol 139: 70-76, 2015.
2. Morice P, Leary A, Creutzberg C, Abu-Rustum N and Darai E: Endometrial cancer. Lancet 387: 1094-1108, 2016.

3. Cui Z, An X, Li J, Liu Q and Liu W: LncRNA MIR22HG negatively regulates miR-141-3p to enhance DAPK1 expression and inhibits endometrial carcinoma cells proliferation. Biomed Pharmacother 104: 223-228, 2018

4. Fatica A and Bozzoni I: Long non-coding RNAs: New players in cell differentiation and development. Nat Rev Genet 15: 7-21, 2014

5. Lin Z, Zhou Z, Guo H, He Y, Pang X, Zhang X, Liu Y, Ao X, Li P, Wang J, et al: Long noncoding RNA gastric cancer-related lncRNA1 mediates gastric malignancy through miRNA-885-3p and cyclin-dependent kinase 4. Cell Death Dis 9: 607, 2018.

6. Quinn JJ and Chang HY: Unique features of long non-coding RNA biogenesis and function. Nat Rev Genet 17: 47-62, 2016.

7. Zhu P, Wang Y, Wu J, Huang G, Liu B, Ye B, Du Y, Gao G, Tian Y, He L and Fan Z: LncBRM initiates YAP1 signalling activation to drive self-renewal of liver cancer stem cells. Nat Commun 7: 13608, 2016.

8. Zhou W, Wang L, Miao Y and Xing R: Novel long noncoding RNA GACAT3 promotes colorectal cancer cell proliferation, invasion and migration through miR-149. Onco Targets Ther 11: 1543-1552, 2018.

9. Cen C, Li J, Liu J, Yang M, Zhang T, Zuo Y, Lin C and Li X: Long noncoding RNA LINC01510 promotes the growth of colorectal cancer cells by modulating MET expression. Cancer Cell Int 18: 45, 2018.

10. Zhang K, Zhao Z, Yu J, Chen W, Xu Q and Chen L: LncRNA FLVCR1-AS1 acts as miR-513c sponge to modulate cancer cell proliferation, migration and invasion in hepatocellular carcinoma. J Cell Biochem 119: 6045-6056, 2018.

11. Guo E, Liang C, He X, Song G, Liu H, Lv Z, Guan J, Yang D and Zheng J: Long Noncoding RNA LINC00958 Accelerates Gliomagenesis Through Regulating miR-203/CDK2. DNA Cell Biol 37: 465-472, 2018.

12. Yang T, Zhai H, Yan R, Zhou Z, Gao L and Wang L: lncRNA CCAT1 promotes cell proliferation, migration and invasion by down-regulation of miR-143 in FTC-133 thyroid carcinoma cell line. Braz J Med Biol Res 51: e7046, 2018.

13. Li Y, Jing F, Ding Y, He Q, Zhong Y and Fan C: Long noncoding RNA CCAT1 polymorphisms are associated with the risk of colorectal cancer. Cancer Genet 222-223: 13-19, 2018.

14. Lai XJ and Cheng HF: LncRNA colon cancer-associated transcript 1 (CCAT1) promotes proliferation and metastasis of ovarian cancer via miR-1290. Eur Rev Med Pharmacol Sci 22: 322-328, 2018.

15. Li Y,Zhu G, Ma Y and Qu H: LncRNA CCAT1 contributes to the growth and invasion of gastric cancer via targeting miR-219-1. J Cell Biochem, 2017 (Epub ahead of print).

16. Chen L, Hu N, Wang C, Zhao H and Gu Y: Long non-coding RNA CCAT1 promotes multiple myeloma progression by acting as a molecular sponge of miR-181a-5p to modulate HOXA1 expression. Cell Cycle 17: 319-329, 2018.

17. Lai Y, Chen Y, Lin Y and Ye L: Down-regulation of LncRNA CCAT1 enhances radiosensitivity via regulating miR-148b in breast cancer. Cell Biol Int 42: 227-236, 2018.

18. Livak KJ and Schmittgen TD: Analysis of relative gene expression data using real-time quantitative PCR and the 2(-Delta Delta C(T)) method. Methods 25: 402-408, 2001.

19. Korhan P, Erdal E and Atabey N: MiR-181a-5p is downregulated in hepatocellular carcinoma and suppresses motility, invasion and branching-morphogenesis by directly targeting c-Met. Biochem Biophys Res Commun 450: 1304-1312, 2014.

20. Prensner JR and Chinnaiyan AM: The emergence of IncRNAs in cancer biology. Cancer Discov 1: 391-407, 2011.

21. Zhang MH, Yang Y, Zhao Y, Wei HB, Ma YQ, Yang CJ, Zhang XJ and Sun YL: LncRNA DQ786243 expression as a biomarker for assessing prognosis in patients with gastric cancer. Eur Rev Med Pharmacol Sci 22: 2304-2309, 2018.

22. Wang YH, Liu YH, Ji YJ, Wei Q and Gao TB: Upregulation of long non-coding RNA HNF1A-AS1 is associated with poor prognosis in urothelial carcinoma of the bladder. Eur Rev Med Pharmacol Sci 22: 2261-2265, 2018.

23. Yang H, Wang S, Kang YJ, Wang C, Xu Y, Zhang Y and Jiang Z: Long non-coding RNA SNHG1 predicts a poor prognosis and promotes colon cancer tumorigenesis. Oncol Rep 40: 261-271, 2018.

24. Li W, Dou Z, We S, Zhu Z, Pan D, Jia Z, Liu H, Wang X and Yu G: Long noncoding RNA BDNF-AS is associated with clinical outcomes and has functional role in human prostate cancer. Biomed Pharmacother 102: 1105-1110, 2018. 
25. Xiao Z, Qu Z, Chen Z, Fang Z, Zhou K, Huang Z, Guo X and Zhang Y: LncRNA HOTAIR is a prognostic biomarker for the proliferation and chemoresistance of colorectal cancer via MiR-203a-3p-mediated Wnt/ $\beta$-catenin signaling pathway. Cell Physiol Biochem 46: 1275-1285, 2018.

26. Cao Y, Shi H, Ren F, Jia Y and Zhang R: Long non-coding RNA CCAT1 promotes metastasis and poor prognosis in epithelial ovarian cancer. Exp Cell Res 359: 185-194, 2017.

27. Zhang Y and Hu H: Long non-coding RNA CCAT1/miR-218/ZFX axis modulates the progression of laryngeal squamous cell cancer. Tumour Biol 39: 1010428317699417, 2017.

28. Zhao J and Cheng L: Long non-coding RNA CCAT1/miR-148a axis promotes osteosarcoma proliferation and migration through regulating PIK3IP1. Acta Biochim Biophys Sin (Shanghai) 49: 503-512, 2017

29. Arunkumar G, Murugan AK, Prasanna Srinivasa Rao H, Subbiah S, Rajaraman R and Munirajan AK: Long non-coding RNA CCAT1 is overexpressed in oral squamous cell carcinomas and predicts poor prognosis. Biomed Rep 6: 455-462, 2017.

30. Tay Y, Rinn J and Pandolfi PP: The multilayered complexity of ceRNA crosstalk and competition. Nature 505: 344-352, 2014.

31. Xu D, Yu J, Gao G, Lu G, Zhang Y and Ma P: LncRNA DANCR functions as a competing endogenous RNA to regulate RAB1A expression by sponging miR-634 in glioma. Biosci Rep 38: pii BSR20171664, 2018.

32. Zhu J, Shi H, Liu H, Wang X and Li F: Long non-coding RNA TUG1 promotes cervical cancer progression by regulating the miR-138-5p-SIRT1 axis. Oncotarget 8: 65253-65264, 2017.
33. Chen Z, Wu J, Huang W, Peng J, Ye J, Yang L, Yuan Y, Chen C, Zhang C, Cai S, et al: Long non-coding RNA RP11-789C1.1 suppresses epithelial to mesenchymal transition in gastric cancer through the RP11-789C1.1/MiR-5003/E-cadherin axis. Cell Physiol Biochem 47: 2432-2444, 2018.

34. Feng L, Shi L, Lu YF, Wang B, Tang T, Fu WM, He W, Li G and Zhang JF: Linc-ROR promotes osteogenic differentiation of mesenchymal stem cells by functioning as a competing endogenous RNA for miR-138 and miR-145. Mol Ther Nucleic Acids 11: 345-353, 2018.

35. Li S, Yang J, Xia Y, Fan Q and Yang KP: Long Noncoding RNA NEAT1 Promotes proliferation and invasion via targeting miR-181a-5p in non-small cell lung cancer. Oncol Res 26: 289-296, 2018.

36. Liu Z, Sun F, Hong Y, Wang B, Tang T, Fu WM, He W, Li G and Zhang JF: MEG2 is regulated by miR-181a-5p and functions as a tumour suppressor gene to suppress the proliferation and migration of gastric cancer cells. Mol Cancer 16: 133, 2017.

37. Yang M,Zhai X, Ge T, Yang C and Lou G: MiR-181a-5p promotes proliferation and invasion and inhibits apoptosis of cervical cancer cells via regulating inositol polyphosphate-5-phosphatase A (INPP5A). Oncol Res 26: 703-712, 2017.

38. Yang CH, Zhang XY, Zhou LN, Wan Y, Song LL, Gu WL, Liu R, Ma YN, Meng HR, Tian YL and Zhang Y: LncRNA SNHG8 participates in the development of endometrial carcinoma through regulating c-MET expression by miR-152. Eur Rev Med Pharmacol Sci 22: 1629-1637, 2018. 